\title{
NUEVOS DATOS SOBRE LA FAUNA CORALINA (SCLERACTINIA) DE LA PLATAFORMA CARBONATADA DE SANT CORNELI, UNIDAD CENTRAL SURPIRENAICA (CRETÁCICO SUPERIOR, SANTONIENSE)
}

\author{
F. Xavier VALLDEPERAS y Eulàlia GILI \\ Departament de Geologia, Universitat Autònoma de Barcelona, Bellaterra 08193.
}

\begin{abstract}
Valldeperas, F.X. y Gili, E. 1999. Nuevos datos sobre la fauna coralina (Scleractinia) de la plataforma carbonatada de Sant Corneli, unidad central surpirenaica (Cretácico Superior, Santoniense). [New data on the coral fauna (Scleractinia) of the Sant Corneli carbonate platform, southern Central Pyrenees (Upper Cretaceous, Santonian)]. Revista Española de Paleontología, $\mathbf{n}^{\mathbf{0}}$ extr. Homenaje al Prof. J. Truyols, 143-159. ISSN 0213-6937.
\end{abstract}

\begin{abstract}
Santonian (Upper Cretaceous) deposits of the Sant Corneli carbonate platform, in the southern Central Pyrenees, contain diverse and abundant corals, besides rudists. Here we present the results of a taxonomic study of the scleractinian coral fauna in the rudist and coral unit of the Sant Martí de Vilanoveta section in the northern flank of the Sant Corneli anticline near Tremp. Twenty-one species from sixteen genera, in eleven different families, are described, of which five species are left in open nomenclature. The great majority of the species recognised are present in, and were originally described from, the Upper Cretaceous Gosau Group and related deposits in the calcareous Alps.

In this work, the species have been identified according the classic taxonomic criteria, as the first stage in an in-depth taxonomic revision of Upper Cretaceous scleractinian corals.
\end{abstract}

Keywords: Corals, Scleractinia, taxonomy, Upper Cretaceous (Santonian), Pyrenees.

\section{RESUMEN}

Los depósitos santonienses (Cretácico Superior) de la plataforma carbonatada de Sant Corneli, en la unidad central surpirenaica contienen, además de rudistas, corales diversos y abundantes. Presentamos los resultados del estudio taxonómico de la fauna de corales escleractinios de la unidad de rudistas y corales de la sección de Sant Martí de Vilanoveta, en el flanco norte del anticlinal de Sant Corneli cerca de Tremp. Se han descrito 21 especies de 16 géneros y 11 familias diferentes; 5 de las especies se dejaron en nomenclatura abierta. La gran mayoría de las especies reconocidas se encuentran y fueron originariamente descritas en el Cretácico Superior del Grupo Gosau y depósitos afines en los Alpes calcáreos.

En este trabajo, las especies han sido identificadas siguiendo un análisis taxonómico clásico, como primer paso a una revisión profunda de la taxonomía de los corales escleractinios del Cretácico Superior.

Palabras clave: Corales, Scleractinia, taxonomía, Cretácico Superior (Santoniense), Pirineos.

\section{INTRODUCCIÓN}

Una de las características más notables de las plataformas carbonatadas de los mares del Tetis en el Cretácico Superior fue el gran desarrollo de comunidades bentónicas dominadas por rudistas, con o sin corales asociados, que dieron lugar a extensas formaciones de rudistas y a formaciones mixtas de rudistas y corales (Masse y Philip, 1981; Gili et al., 1995; Skelton et al., 1997; Sanders y Baron-Szabo, 1997; Stössel, 1997). A pesar de que hace más de un siglo que los corales del Cretácico Superior del Grupo Gosau (Alpes calcáreos) son universalmente conocidos y que la fauna coralina de este periodo es muy diversa (Rosen, en prensa), la presencia de corales en facies carbonatadas tropicales del Cretácico Superior ha sido muy poco explorada y, a menudo, omitida. Esta arbitrariedad puede haberse debido a la pobre conservación que generalmente presentan los corales y a la confusión reinante en la taxonomía de los corales escleractinios del Cretácico Superior.

Los depósitos de plataforma carbonatada del Santoniense del anticlinal de Sant Corneli en la unidad central surpirenaica contienen, además de rudistas, corales diversos y abundantes. Del análisis bibliográfico se desprende que a pesar del progreso realizado en el conocimiento de los ambientes deposicionales carbonatados y faunas de dicha plataforma, especial- 


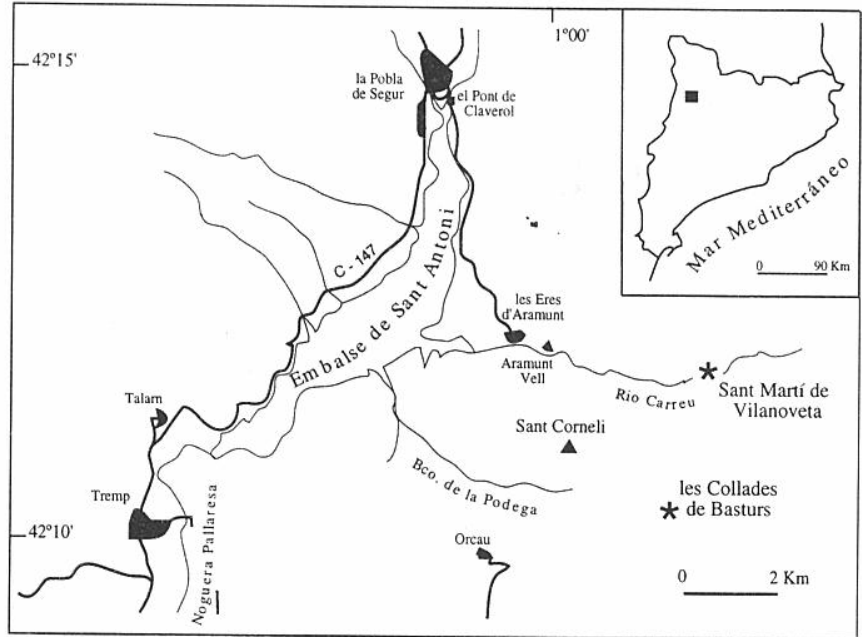

Figura 1. Situación geográfica de las secciones de Sant Martí de Vilanoveta y les Collades de Basturs en la comarca catalana del Pallars Jussà.

mente de los rudistas (Gili, 1984, 1992; Ross, 1989; Skelton et al., 1995; Gili et al., 1996), los corales han estado relativamente olvidados. Desde que J. R. Bataller (1937, 1945) elaboró una serie de listas de géneros y especies que, en algunos casos, describió brevemente, no se conoce ningún trabajo taxonómico relevante hasta Asunción Vidal (1980) que describe y determina parte de la fauna coralina de les Collades de Basturs en la vertiente sur de la sierra de Sant Corneli. El objetivo de - este artículo es presentar los resultados del análisis taxonómico preliminar de la fauna coralina de la plataforma de Sant Corneli. El estudio se ha centrado principalmente en las asociaciones de corales de la 'unidad de rudistas y corales' de la sección de Sant Martí de Vilanoveta, en el flanco norte del anticlinal de Sant Corneli (ver Gili et al., 1996), completándose el estudio con algunas muestras recogidas en les Collades de Basturs (Fig. 1) (ver Gili et al., 1996 para la situación geológica de ambas secciones).

Se recogieron un total de 134 muestras coralinas, 122 en Sant Martí de Vilanoveta y 12 en les Collades de Basturs, la mayoría procedentes de la biofacies de corales planos (Skelton et al., 1997; Valldeperas, 1997). En Sant Martí de Vilanoveta las muestras se cogieron en 11 series estratigráficas detalladas (escala $\mathrm{cm}$ ), de $11 \mathrm{~m}$ de potencia máxima y lateralmente correlacionables en el campo, así como en 11 transectos continuos. (Ver Valldeperas, 1997 para la situación y descripción de los afloramientos.)

La mayoría de ejemplares fueron preparados (secciones pulidas y láminas delgadas transversales y longitudinales) para su análisis en el microscopio óptico, lo que permitió el estudio detallado de la microestructura de los elementos radiales y de la arquitectura interna de la colonia, caracteres éstos en los que se basa principalmente la taxonomía de los corales escleractinios. Las 134 muestras recogidas han proporcionado 66 determinaciones, el resto de ejemplares no ha podido ser determinado debido a su pobre estado de conservación.

\section{ANÁLISIS TAXONÓMICO}

Las determinaciones taxonómicas han estado sujetas al estado de conservación de los fósiles. Ninguno de los corales estudiados conserva la mineralogía original que ha sido reemplazada por calcita neomórfica. En la mayoría de los casos, la superficie calicinal no se ha conservado, pero parte de la morfología del esqueleto se ha conservado gracias a la formación de envueltas micríticas en los elementos estructurales y al relleno de sedimento de la porosidad intraesquelética en la zona periférica de la colonia. Los elementos estructurales así conservados presentan a menudo un contorno difuso, lo que dificulta la medición exacta de su anchura y longitud. Finalmente, algunos corales están parcial o totalmente silicificados, lo que implica una modificación importante de la morfología y dimensiones de los diferentes elementos esqueléticos en las zonas superficiales, y la pérdida de una gran parte del esqueleto interno.

La clasificación utilizada para la determinación genérica y específica de los corales es la propuesta por Alloiteau (1952, 1957), ampliada y modificada por Beauvais (1982). Para los niveles taxonómicos supragenéricos se ha utilizado la clasificación más ampliamente aceptada en la literatura, la propuesta por Beauvais (1980) y corroborada por Morycowa y Roniewicz (1995) y que es compatible con las de Veron (1995) y Veron et al. (1996), basadas en estudios moleculares.

Los ejemplares estudiados han sido registrados y depositados en la colección de paleontología del Departamento de Geología de la Universidad Autónoma de Barcelona. En el subapartado 'Material' de los apartados dedicados a las especies en este capítulo, se ha incluido sólo el número de registro de los ejemplares figurados en este artículo o en Valldeperas (1997).

ORDEN SCLERACTINIA Bourne, 1900

SUBORDEN ARCHAEOCAENIINA Alloiteau, 1952, emend. Beauvais, 1980

Familia Astrocoeniidae Koby, 1890 Actinastraea d'Orbigny, 1849

Especie tipo: Astrea goldfussi d'Orbigny, 1849.

Actinastraea piveteaui Alloiteau, 1954

Fig. 3.E

*1954 Actinastrea piveteaui Alloiteau, 87, lám. X, fig. 5.

Material: Una colonia de hábito domal (UAB-32180), de les Collades de Basturs; y tres colonias, dos de hábito laminar (UAB-32181, UAB-32182) y una domal (UAB32183), de Sant Martí de Vilanoveta.

\section{Descripción}

Colonia cerioide, ocasionalmente plocoide (i. e. delgada periteca de costillas cortas entre 4-5 poliporitos). Cálices circulares en los poliporitos adultos y poligonales (comúnmente pentagonales) en los juveniles. Presencia de 24 elementos radiales, septos en las disposiciones cerioides y costoseptos en las plocoides, distribuidos en dos ciclos. 


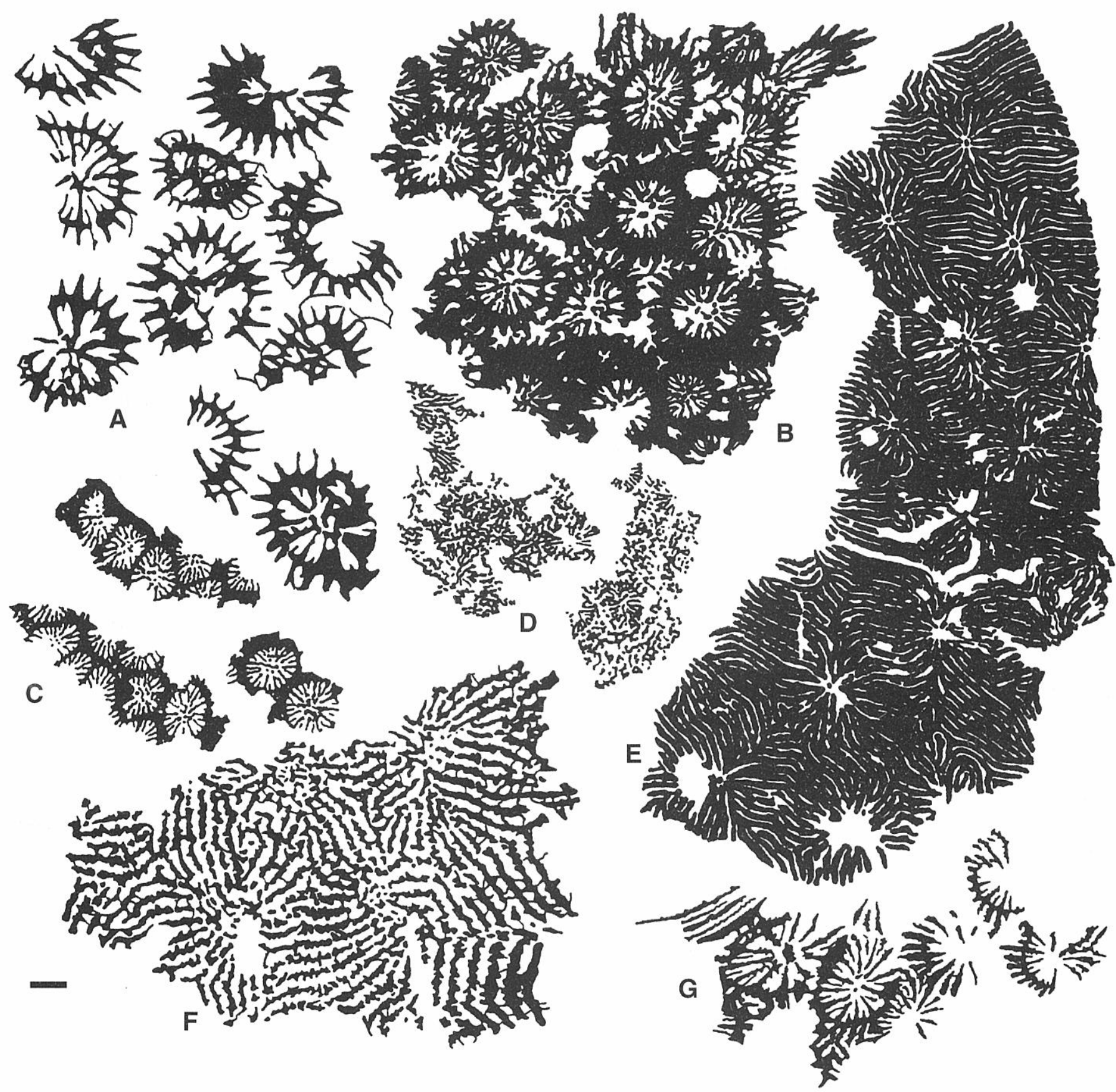

Figura 2. Dibujos realizados a partir de la proyección de láminas delgadas; en negro se ha representado el esqueleto. Los ejemplares, si no se indica otra cosa, proceden de Sant Martí de Vilanoveta y están depositados en la colección de paleontología del Departamento de Geología de la Universidad Autónoma de Barcelona. (Escala gráfica: $1 \mathrm{~mm}$.) Este párrafo se aplica también a las Figs. 3 - 5.

A. Placocoenia decussata (Oppenheim, 1930). Ejemplar UAB-32156. Sección transversal. Amplia periteca con disepimentario exotecal formado por grandes y escasos disepimentos. B. Columastrea sp. Ejemplar UAB-32171. Sección transversal. C. Columactinastraea? sp. Ejemplar UAB-32175. Sección transversal. D. Actinacis haueri Reuss, 1854. Ejemplar UAB-32189. Sección transversal. La exoteca reticular presenta una estructura caótica que impide la clara distinción de los poliporitos apenas circunscritos por una muralla perforada y discontinua. E. Thamnasteria aff. andranomarivensis Alloiteau, 1958. Ejemplar UAB-32166. Sección transversal. La anastomosis de los elementos radiales es muy regular, caracterizada por la formación de 2 grupos de 2 elementos radiales, 6 grupos de 4, 5 o 6 elementos, quedando siempre 2 elementos libres. F. Brachyseris tenerrima (Oppenheim, 1930). Ejemplar UAB-32185. Sección transversal. G. Neocaeniopsis fromenteli Beauvais, 1982. Ejemplar UAB-32174. Sección transversal.

Los elementos radiales de primer ciclo son continuos, los septos ensanchados (en el margen costal) y los costoseptos bicuneiformes. Los de segundo ciclo, mucho más delgados que los del primero, tienen comúnmente forma de zigzag 
(forma también observada en algunos elementos radiales de primer ciclo) debido a la ornamentación que presentan sus caras laterales (gránulos espiniformes alternos, grandes, progresivamente más desarrollados hacia el margen axial). Elementos radiales subconfluentes, la mayoría libres (algunos elementos de primer ciclo se unen en el eje axial). Columnilla estiliforme gruesa, elíptica y en algunos poliporitos ligeramente laminar. En este último caso, el eje mayor de la columnilla se dispone según el plano que forman dos elementos radiales de primer ciclo. Presencia de algunos disepimentos gruesos que unen principalmente elementos radiales de segundo ciclo con elementos radiales de primer ciclo por su margen axial. Muralla compacta y continua. Gemación intracalicinal e intercalicinal. (Ver las dimensiones de los caracteres descritos, en la Tabla 1.)

\section{Columactinastraea Alloiteau, 1952}

Especie tipo: Columactinastraea rennensis Alloiteau, 1952.

\section{Columactinastraea? sp.}

Fig. 2.C

Material: Colonia de hábito laminar (UAB-32175), de Sant Martí de Vilanoveta.

\section{Descripción}

Colonia cerioide, ocasionalmente plocoide en los puntos de unión de diversos poliporitos. Cálices tetragonales o pentagonales, con 24 septos distribuidos en tres ciclos. Los septos son moderadamente claviformes, continuos, confluentes y libres. Sus caras laterales presentan algunos gránulos espiniformes, menos frecuentemente redondeados, en posición alterna a ambos lados del septo. Columnilla estiliforme rodeada por una corona de 6 pali que corresponden a los septos de segundo ciclo. Endoteca disepimental escasa y localizada en la región axial. Muralla septotecal compacta y continua, formada por la intercalación de estereomas entre los septos en la región periférica de cada poliporito o por el ensanchamiento de los septos. Algunas lagunas (pequeñas zonas sin esclerénquima) entre los poliporitos. (Ver las dimensiones de los caracteres descritos, en la Tabla 1.)

\section{Observaciones}

Este género, según su autor, tiene generalmente 3 ciclos (simetría 6: $6_{1}+6_{2}+12_{3}=24$ ); sin embargo, la especie tipo tiene 2 ciclos (simetría 8: $8_{1}+8_{2}=16$ ).

SUBORDEN ARCHAEOFUNGIINA Alloiteau, 1952,
emend. Beauvais, 1980

Familia Cyclophyllopsiidae Alloiteau, 1952, emend. Beauvais, 1982

Placocoenia d'Orbigny, 1849

Especie tipo: Astrea macrophtalma Goldfuss, 1826.

Placocoenia dumortieri de Fromentel, 1879 Fig. 5.E
.1982 Placocoenia dumortieri de Fromentel; Beauvais, v. 1, 107; y su sinonimia.

Material: Colonia de hábito tábulo-lenticular (UAB-32155), de Sant Martí de Vilanoveta.

\section{Descripción}

Colonia plocoide con cálices circulares o ligeramente elípticos, con 24 costoseptos distribuidos en tres ciclos. Los costoseptos son bicuneiformes, con la región costal mucho más gruesa y la mitad de larga que la región septal (los de primer ciclo también pueden ser claviformes o ropaloides), continuos, no confluentes, ocasionalmente subconfluentes. Las caras laterales de los costoseptos presentan un número variable de gránulos alternos, espiniformes, bastante e irregularmente pronunciados. Columnilla laminar delgada. Endoteca disepimental vesicular abundante y muralla septotecal compacta y continua. Exoteca disepimental vesicular, con disepimentos grandes y muy curvados. Gemación extracalicinal. (Ver las dimensiones de los caracteres descritos, en la Tabla 1.)

\section{Observaciones}

La mayoría de los caracteres del ejemplar de Sant Martí de Vilanoveta coinciden con los de Placocoenia dumortieri, pero se diferencia de la descripción que Beauvais (1982) hace de esta especie por presentar disepimentos exotecales grandes y muy curvados en vez de finos y celulares.

\section{Placocoenia decussata (Oppenheim, 1930)}

Fig. 2.A

*1930 Nefocoenia decussata Oppenheim, 421, lám. XXXIX, figs. 2-4.

non 1937 Nefocoenia decusata Oppenheim; Bataller, 103.

.1982 Placocoenia decussata (Oppenheim); Beauvais, v. 1,110 .

Material: Colonia de hábito domal (UAB-32156), de Sant Martí de Vilanoveta.

\section{Descripción}

Colonia plocoide de cálices circulares o ligeramente elípticos. En este último caso, el diámetro mayor de la elipse coincide con el plano de la columnilla laminar. Los cálices sobresalen bastante, así en la sección transversal más superficial no se observa la robusta exoteca que presentan las secciones más profundas u oblicuas. Presencia de 24 costoseptos por poliporito distribuidos en tres ciclos. Los del primero y segundo ciclos son bicuneiformes o claviformes, mientras que los del tercero son siempre bicuneiformes y más cortos que los anteriores. Son costoseptos continuos y no confluentes, y presentan algunos gránulos espiniformes, tanto en la región septal como en la costal. Los costoseptos de primer ciclo, y a veces también del segundo, pueden estar unidos entre sí (uniones dobles o triples) o entre sí y la columnilla por medio de disepimentos gruesos, mientras que los de tercer ciclo son libres. Columnilla laminar orientada según el plano que forman dos elementos radiales de primer ciclo. Muy pocos disepimentos endotecales y muchos 


\begin{tabular}{|c|c|c|c|c|c|c|c|c|c|c|c|c|c|c|c|c|c|c|c|c|c|c|}
\hline 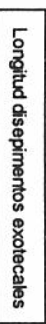 & 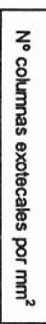 & 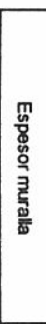 & 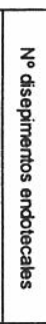 & 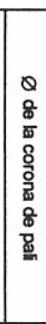 & $\begin{array}{l}Q \\
0 \\
0 \\
\overline{0} \\
0 \\
\mathbb{D}\end{array}$ & 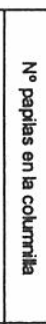 & 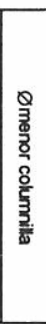 & 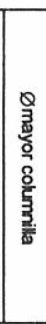 & 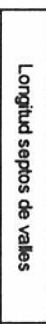 & 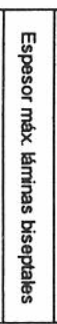 & 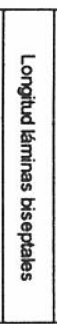 & 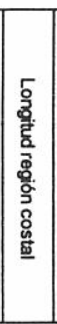 & 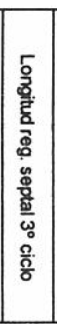 & 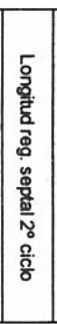 & 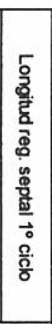 & 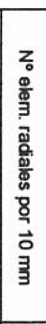 & 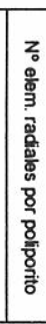 & 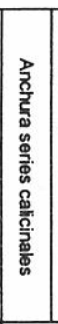 & 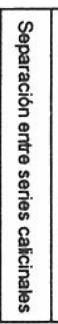 & 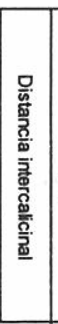 & 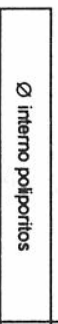 & \\
\hline 1 & 1 & $\stackrel{0}{N}$ & 1 & 1 & 1 & 1 & 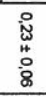 & 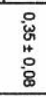 & 1 & ' & 1 & $\begin{array}{l}\stackrel{8}{\alpha} \\
\stackrel{4}{0} \\
\stackrel{0}{0}\end{array}$ & 1 & 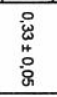 & 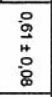 & 1 & $\simeq$ & 1 & 1 & $\begin{array}{l}\vec{\Psi} \\
\stackrel{\leftrightarrow}{\leftrightarrow} \\
\stackrel{\leftrightarrow}{\Psi} \\
\end{array}$ & 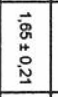 & Actinastraea piveteaui \\
\hline 1 & 1 & 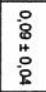 & $\ddot{\square}$ & 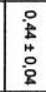 & $\begin{array}{l}\circ \\
\stackrel{\circ}{0} \\
\circ \\
\end{array}$ & 1 & 1 & $\begin{array}{l}\circ \\
8 \\
8 \\
0 \\
0\end{array}$ & ' & 1 & ' & 1 & 1 & 1 & 1 & 1 & $\simeq$ & 1 & 1 & 1 & 1 & Columactinastraea ? sp. \\
\hline 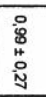 & 1 & 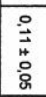 & $\stackrel{n}{\square}$ & ' & 1 & 1 & 1 & 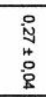 & 1 & ' & 1 & 1 & 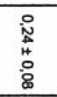 & $\begin{array}{l}0 \\
8 \\
4 \\
4 \\
8 \\
8\end{array}$ & 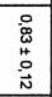 & 1 & $\simeq$ & 1 & ' & 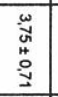 & 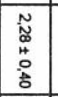 & Placocoenia dumortieri \\
\hline 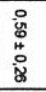 & 1 & 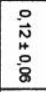 & 1 & 1 & 1 & 1 & 1 & 1 & 1 & 1 & 1 & $\begin{array}{l}\circ \\
\dot{8} \\
\stackrel{4}{0} \\
\dot{\vec{\omega}} \\
\end{array}$ & \begin{tabular}{l}
0 \\
\multirow{1}{*}{} \\
$ث$ \\
0 \\
0
\end{tabular} & $\begin{array}{l}\circ \\
\stackrel{8}{8} \\
4 \\
0 \\
\dot{\vec{\sigma}}\end{array}$ & 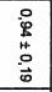 & 1 & $\simeq$ & 1 & ' & 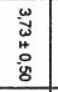 & 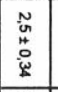 & Placocoenia decussata \\
\hline 1 & 1 & ' & 1 & 1 & 1 & 1 & 1 & 1 & 1 & 1 & 1 & 1 & 1 & 1 & 1 & $\underset{N}{N}$ & $\omega$ & 1 & 1 & 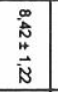 & 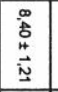 & $\begin{array}{l}\text { Corbariastraea } \\
\text { junctiseptata }\end{array}$ \\
\hline 1 & 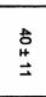 & 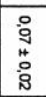 & 1 & 1 & ' & 1 & 1 & $\begin{array}{l}\circ \\
\stackrel{8}{8} \\
\stackrel{4}{\circ} \\
\stackrel{3}{3}\end{array}$ & 1 & 1 & 1 & 1 & 1 & 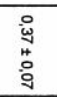 & 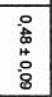 & 1 & $\simeq$ & 1 & 1 & 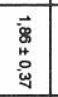 & 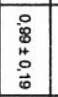 & Actinacis reussi \\
\hline 1 & 1 & 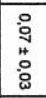 & 1 & 1 & ' & 1 & 1 & 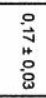 & 1 & 1 & 1 & 1 & 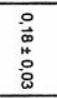 & $\begin{array}{l} \\
\stackrel{\circ}{\vec{\omega}} \\
ث \\
\stackrel{0}{0} \\
\dot{\omega}\end{array}$ & 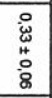 & 1 & $\simeq$ & 1 & 1 & 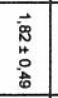 & 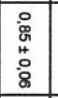 & Actinacis haueri \\
\hline 1 & 1 & 1 & 1 & 1 & ' & 总 & 1 & $\begin{array}{l}\overrightarrow{\vec{\psi}} \\
\stackrel{4}{0} \\
\stackrel{0}{0} \\
\overrightarrow{0}\end{array}$ & 1 & 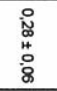 & 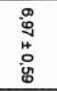 & 1 & 1 & 1 & 1 & 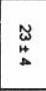 & $\begin{array}{l}\vec{f} \\
\vec{\omega} \\
\vec{\omega}\end{array}$ & 1 & 1 & 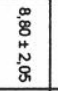 & 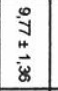 & Astraraea media \\
\hline 1 & 1 & 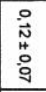 & 1 & 1 & 1 & ' & 1 & 1 & 1 & 1 & 1 & 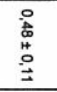 & 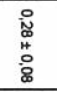 & 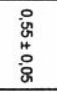 & $\begin{array}{l}\circ \\
\stackrel{8}{8} \\
\stackrel{2}{0} \\
\stackrel{0}{0}\end{array}$ & 1 & $\simeq$ & 1 & 1 & 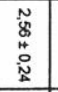 & $\begin{array}{l}\overrightarrow{8} \\
\overrightarrow{8} \\
\stackrel{0}{0} \\
\vec{\omega}\end{array}$ & Neocaeniopsis excelsa \\
\hline 1 & 1 & 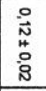 & 1 & 1 & 1 & 1 & 1 & $\stackrel{\circ}{\circ}$ & 1 & 1 & ' & 1 & 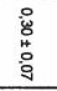 & 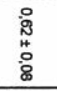 & $\begin{array}{l}.8 \\
\dot{8} \\
\stackrel{4}{0} \\
\stackrel{\vec{\omega}}{\omega}\end{array}$ & 1 & $\simeq$ & 1 & 1 & 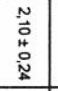 & 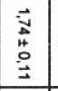 & Neocaeniopsis fromenteli \\
\hline 1 & 1 & 1 & 1 & 1 & 1 & 1 & 1 & $\begin{array}{l}\stackrel{0}{T} \\
\stackrel{4}{*} \\
\stackrel{0}{\circ}\end{array}$ & 1 & 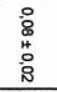 & $\begin{array}{l}\overrightarrow{\overrightarrow{\vec{\omega}}} \\
\stackrel{4}{\circ} \\
\dot{8} \\
\end{array}$ & ' & 1 & 1 & 1 & $\stackrel{\text { 㞻 }}{\sim}$ & 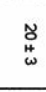 & 1 & 1 & $\begin{array}{l}\overrightarrow{9} \\
\overrightarrow{9} \\
\stackrel{4}{0} \\
\stackrel{\omega}{\omega}\end{array}$ & 1 & Mesomorpha mammillata \\
\hline 1 & 1 & 1 & 1 & 1 & 1 & 1 & 1 & 1 & 1 & $\begin{array}{l}\stackrel{0}{\tilde{N}} \\
\vec{\Delta} \\
\stackrel{0}{0} \\
\end{array}$ & 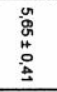 & , & 1 & 1 & 1 & $\stackrel{\mathscr{S}}{4}$ & $\simeq$ & 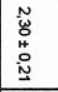 & $\begin{array}{l}\stackrel{n}{0} \\
\stackrel{0}{ \pm} \\
\stackrel{\Xi}{\Xi}\end{array}$ & 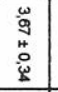 & 1 & Fungiastraea sp. \\
\hline 1 & 1 & 1 & 1 & 1 & I & 1 & 1 & $\begin{array}{l}\stackrel{\circ}{\vec{\omega}} \\
\stackrel{4}{*} \\
\stackrel{0}{\circ}\end{array}$ & 1 & 1 & N & 1 & 1 & 1 & 1 & $\underset{\omega}{\stackrel{s}{\omega}}$ & 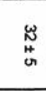 & $\begin{array}{l}\stackrel{\omega}{\vec{J}} \\
\ddot{*} \\
\stackrel{0}{\circ}\end{array}$ & $\begin{array}{l}\tilde{\mathbf{g}} \\
\ddot{8} \\
\stackrel{0}{8} \\
\end{array}$ & 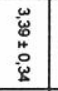 & , & $\begin{array}{l}\text { Thamnasteria aff. } \\
\text { andranomarivensis }\end{array}$ \\
\hline 1 & 1 & 1 & 1 & 1 & I & 1 & 1 & 1 & 1 & 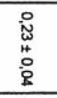 & , & 1 & 1 & 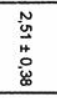 & 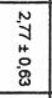 & $\underset{\omega}{\stackrel{\sim}{W}}$ & $\begin{array}{l}\widetilde{W} \\
\stackrel{\sigma}{*}\end{array}$ & $\begin{array}{l}\tilde{\omega} \\
\tilde{N} \\
4 \\
0 \\
\dot{8} \\
\end{array}$ & 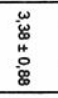 & 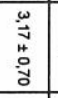 & 1 & Dimorphastraea sp. 1 \\
\hline 1 & 1 & 1 & 1 & 1 & ' & 1 & 1 & 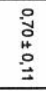 & 1 & 1 & 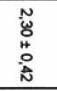 & 1 & 1 & 1 & 1 & $\stackrel{\mathbb{H}}{\stackrel{\sim}{N}}$ & 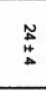 & 1 & 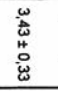 & $\begin{array}{l}\tilde{N} \\
\tilde{W} \\
\stackrel{4}{0} \\
\tilde{\tilde{W}} \\
\end{array}$ & ' & Dimorphastraea sp.2 \\
\hline 1 & 1 & 1 & 1 & 1 & 1 & 1 & 1 & 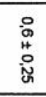 & 1 & 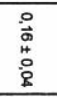 & $\begin{array}{l}\overrightarrow{\vec{\omega}} \\
\stackrel{\vec{\Delta}}{\vec{\Delta}}\end{array}$ & ' & 1 & 1 & 1 & $\stackrel{\mathscr{L}}{\uplus}$ & $\stackrel{\stackrel{\leftrightarrow}{*}}{\sim}$ & ' & 1 & 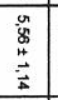 & 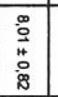 & Valliculastraea spinosa \\
\hline 1 & 1 & 1 & 1 & 1 & 1 & 1 & 1 & 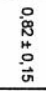 & 1 & 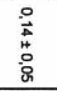 & 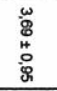 & ' & 1 & 1 & 1 & 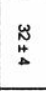 & $\underset{\stackrel{\mathscr{L}}{\overrightarrow{0}}}{\mathrm{a}}$ & 1 & 1 & 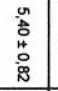 & 1 & Valliculastraea texta \\
\hline 1 & 1 & 1 & 1 & 1 & 1 & $\underset{\stackrel{\vec{\omega}}{\sim}}{\stackrel{\sim}{\sim}}$ & ' & 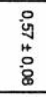 & 1 & 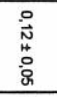 & 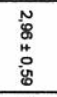 & , & 1 & 1 & 1 & 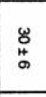 & 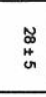 & 1 & 1 & $\begin{array}{l}\omega \\
\stackrel{\omega}{1} \\
4 \\
0 \\
\dot{\sigma} \\
\end{array}$ & ' & Brachyseris tenerrima \\
\hline 1 & 1 & 1 & 1 & 1 & 1 & 1 & 1 & 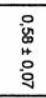 & 1 & $\begin{array}{l} \\
\dot{\vec{H}} \\
\stackrel{0}{0} \\
\dot{\Delta}\end{array}$ & 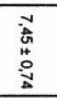 & , & 1 & 1 & 1 & $\underset{\dddot{\#}}{\Psi}$ & $\underset{\stackrel{\vec{\omega}}{\rightleftarrows}}{\vec{\rightleftarrows}}$ & 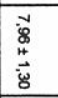 & 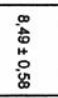 & $\begin{array}{l}\vec{\Delta} \\
\overrightarrow{8} \\
\overrightarrow{\vec{N}}\end{array}$ & 1 & $\begin{array}{l}\text { Elasmofungia } \\
\text { comoserioides }\end{array}$ \\
\hline 1 & ' & 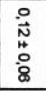 & $\underset{\omega}{\overrightarrow{+}}$ & 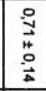 & $\begin{array}{l}\stackrel{0}{1} \\
\overrightarrow{4} \\
\stackrel{0}{\circ} \\
\dot{0}\end{array}$ & 1 & 1 & 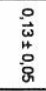 & 1 & 1 & 1 & 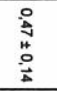 & 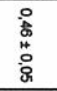 & 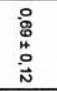 & $\begin{array}{l}0 \\
\stackrel{0}{0} \\
4 \\
0 \\
0\end{array}$ & 1 & $\simeq$ & 1 & 1 & 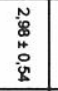 & 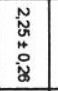 & Columastrea sp. \\
\hline 1 & 1 & 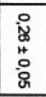 & 1 & 1 & 1 & 1 & 1 & 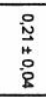 & 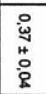 & 1 & I & 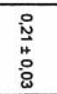 & 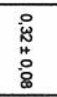 & 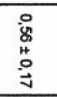 & $\begin{array}{l}\stackrel{0}{y} \\
ث \\
\stackrel{\Delta}{\Delta} \\
\end{array}$ & 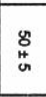 & 1 & $\begin{array}{l}\overrightarrow{\vec{\Delta}} \\
\stackrel{\Delta}{0} \\
\overrightarrow{\vec{\omega}} \\
\end{array}$ & 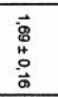 & 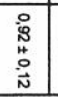 & 1 & Meandroria tenella \\
\hline
\end{tabular}

Tabla 1. Parámetros biométricos (las dimensiones en $\mathrm{mm}$ ) de los ejemplares de Sant Martí de Vilanoveta y les Collades de Basturs. Los valores corresponden a la media muestral (o la moda, en el caso de las variables discretas), seguida de la desviación típica muestral corregida (o de la mitad del rango, en su caso). La omisión de valores es debida a circunstancias tales como el mal estado de los ejemplares, material insuficiente, etc. 
disepimentos exotecales, grandes, robustos y vesiculares. Muralla compacta y continua. Periteca bien desarrollada, constituida por un disepimentario vesicular robusto, y regiones costales cortas, anchas y del mismo tamaño, independientemente del elemento radial de que se trate, excepto en algunos casos en que las regiones costales de los costoseptos de primero y segundo ciclos presentan un desarrollo menor que las del tercero. (Ver las dimensiones de los caracteres descritos, en la Tabla 1.)

\section{Observaciones}

El ejemplar estudiado presenta una distancia entre centros calicinales de poliporitos contiguos menor que la que presenta el holotipo de Placocoenia decussata. Hemos supuesto que esta diferencia podría ser debida a factores ecológicos, basándonos en los resultados obtenidos por diversos autores (e. g. Foster, 1979; 1983; 1985; Budd et al., 1994; Amaral, 1994; Riegl y Piller, 1997) sobre la variabilidad de los corales actuales y fósiles.

Familia Procyclolitidae Vaughan y Wells, 1943, emend. Alloiteau, 1952

Corbariastraea Alloiteau, 1952

Especie tipo: Corbariastraea rennensis Alloiteau, 1952.

\section{Corbariastraea junctiseptata \\ (Oppenheim, 1930)}

Fig. 4.C

*1930 Synastraea (?) junctiseptata Oppenheim, 174, lám. XXVIII, fig. 1-1a.

1982 Corbariastraea junctiseptata (Oppenheim); Beauvais, v. II, 20, lám. XXIII, fig. 2.

Material: Una colonia de hábito pedunculado, parcialmente silicificada (UAB-32165), de les Collades de Basturs y un ejemplar (UAB-32164), de Sant Martí de Vilanoveta.

\section{Descripción}

Colonia thamnasterioide de cálices grandes, circulares, sin márgenes marcados. Las fosetas calicinales son muy poco profundas, excepto en el eje donde se profundizan ligeramente. Presencia de un número elevado de láminas biseptales (43), de sección rectangular y de igual longitud, ligeramente onduladas y compactas. Las caras laterales presentan numerosos gránulos espiniformes, distribuidos regularmente, opuestos a ambos lados de la lámina. Los márgenes distales de los elementos radiales son difíciles de distinguir, pero parecen denticulados, ligeramente heterodontos. Las láminas biseptales pueden presentar anastomosis múltiples irregulares o unirse en el eje sin formar engrosamientos. Ausencia de columnilla. Sinaptículos formados por la extensión de algunos gránulos, sobre todo en la región media de las láminas biseptales. Disepimentos finos, celulares, principalmente en la región axial. Sin muralla, pero con una marcada holoteca que recubre el pedúnculo y la superficie basal de la colonia. (Ver las dimensiones de los caracteres descritos, en la Tabla 1.)
SUBORDEN DENDROPHYLLIINA Vaughan y Wells, 1943

Familia Actinacididae Vaughan y Wells, 1943

Actinacis d'Orbigny, 1849

Especie tipo: Actinacis martiniana d'Orbigny, 1849.

\section{Actinacis reussi Oppenheim, 1930 \\ Fig. 4.E}

*1930 Actinacis reussi Oppenheim, 8, lám. 1, figs. 6-6a; lám. X, figs. 2-2a; lám. XV, fig. 6.

1982 Actinacis reussi Oppenheim; Beauvais, v. II, 271, lám. XLVIII, figs. 5-6.

Material: Cuatro ejemplares (de ellos se ha ilustrado el UAB-32152), de Sant Martí de Vilanoveta.

\section{Descripción}

Colonia plocoide de cálices circulares. Generalmente 24 septos por poliporito (menos comúnmente 22 y ocasionalmente 20) distribuidos en dos ciclos de difícil diferenciación. Septos muy anchos, con poros verticales en la región más axial, no confluentes, comúnmente coalescentes pero también algunos libres. En los septos coalescentes, la anastomosis puede ser simple o múltiple (triple) e irregular y puede tener lugar entre septos contiguos o entre septos separados por otro elemento radial; la unión puede ser directa o por medio de un sinaptículo. En el primer caso, si la sección transversal corta los septos por los poros, la unión de los septos, en forma de $\mathrm{V}$ o de $\mathrm{Y}$, queda separada del resto. Columnilla estiliforme. Sinaptículos abundantes en la zona axial del poliporito. Muralla sinapticulotecal perforada y discontinua. Exoteca tábulo-columnar densa (de aspecto granular en sección transversal). (Ver las dimensiones de los caracteres descritos, en la Tabla 1.)

\section{Observaciones}

$\mathrm{La}$ anastomosis en $\mathrm{V}$ o en $\mathrm{Y}$ de los márgenes axiales de los elementos radiales perforados de Actinacis ha ocasionado numerosas confusiones. Así, Alloiteau (1952) considera los márgenes axiales como una corona de pali, y Wells (1956) como una columnilla parietal fascicular. Por otra parte, la distancia entre centros calicinales contiguos en los ejemplares estudiados difiere ligeramente de la presentada por Beauvais (1982) para los ejemplares que atribuye a Actinacis reussi (2-3 mm frente a los 1,46-2,26 mm de nuestros ejemplares).

\section{Actinacis haueri Reuss, 1854}

Fig. 2.D

1982 Actinacis haueri Reuss; Beauvais, v. II, 268, lám. XLVIII, fig. 3 ; y su sinonimia.

Material: Colonia de hábito ramoso delicado (UAB-32189), de Sant Martí de Vilanoveta.

\section{Descripción}

Colonia plocoide de cálices circulares, con 24 septos por poliporito distribuidos en tres ciclos difíciles de distinguir 


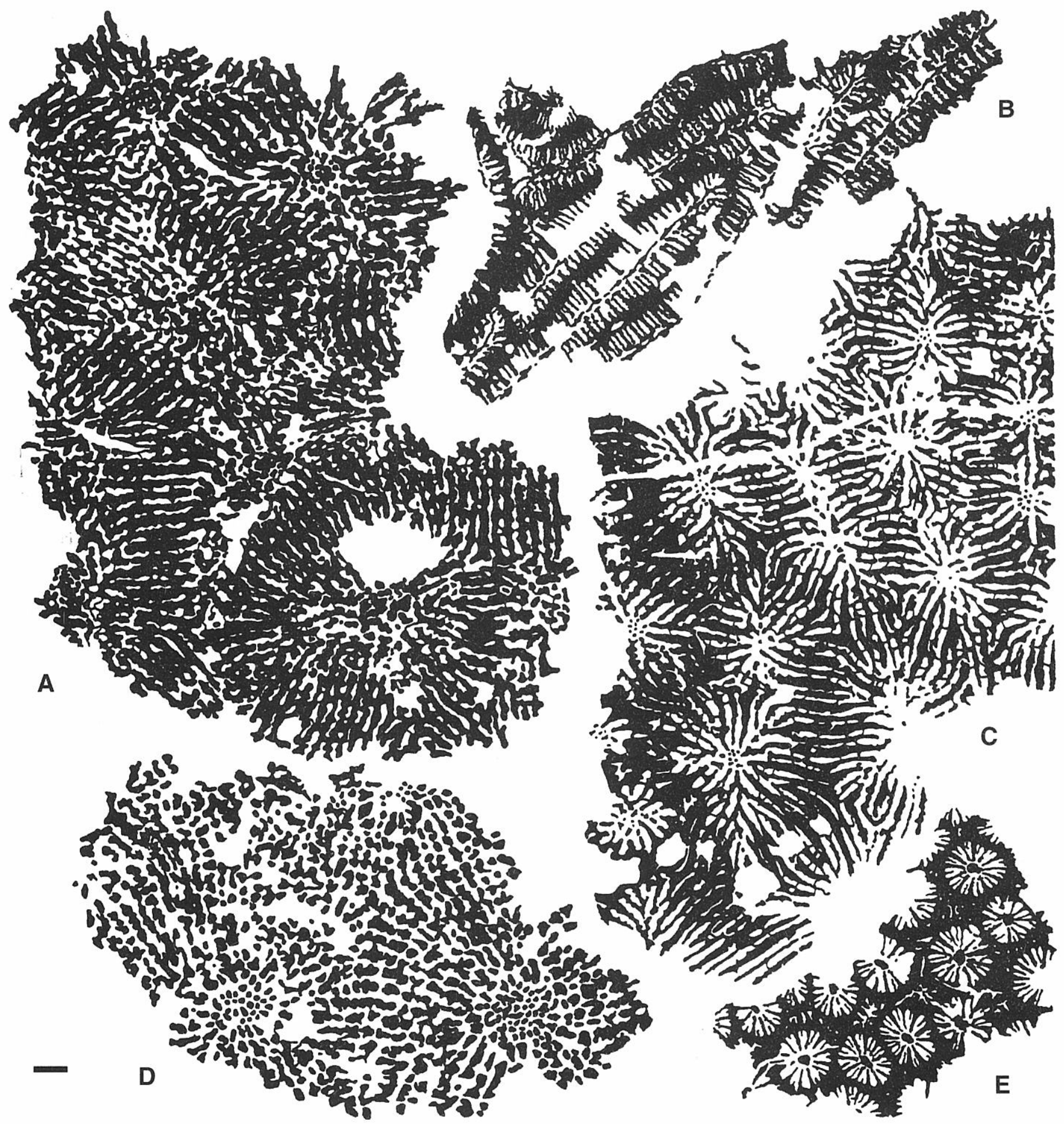

Figura 3. (Véase la leyenda de la Fig. 2.)

A. Valliculastraea texta (Oppenheim, 1930). Ejemplar UAB-32157. Sección transversal. B. Meandroria tenella (Goldfuss, 1826). Ejemplar UAB-32150. Sección transversal. En una de las series calicinales (inferior izquierda) se observan claramente los tres ciclos de elementos radiales. C. Dimorphastraea sp. 1. Ejemplar UAB-32167. Sección transversal. D. Astraraea media (Sowerby, 1835). Ejemplar UAB-32177. Sección transversal. E. Actinastraea piveteaui Alloiteau, 1954. Ejemplar UAB-32181. Sección transversal. Se reconocen zonas de desenganche mural en las que se pueden observar las regiones costales de los elementos radiales y la ausencia de elementos exotecales.

debido a que los septos de segundo y tercer ciclos tienen una longitud muy parecida. Septos anchos, continuos, que ocasionalmente presentan perforaciones en su margen axial. Caras laterales con algún grano espiniforme muy poco desarrollado y en disposición alterna. Septos no confluentes, con frecuentes anastomosis irregulares que pueden formarse tanto por la unión del margen axial de un septo de tercer ciclo con las caras laterales de dos septos de segundo ciclo 
(uniones triples), como por la unión de septos de segundo ciclo con septos del primero. Presencia de una pequeña columnilla parietal papilar. Algún sinaptículo, confinado principalmente en la región axial y algún disepimento endotecal celular muy fino, en la región periférica del poliporito. Muralla sinapticulotecal perforada y discontinua. Exoteca reticulada ("vermiculada" en Beauvais, 1982), de aspecto esponjoso en sección transversal, formada por la unión de trabéculas, sinaptículos gruesos y tábulas discontinuas. (Ver las dimensiones de los caracteres descritos, en la Tabla 1.)

\section{Observaciones}

Beauvais (1982) utiliza la densidad trabecular (número de trabéculas por $\mathrm{mm}^{2}$ ) como carácter taxonómico principal para diferenciar especies en Actinacis. En el ejemplar estudiado, la densidad trabecular no ha podido ser calculada debido a que las características, por otra parte propias, de la exoteca "vermiculada" han imposibilitado la individualización de las trabéculas.

\section{Familia Astraraeidae Beauvais, 1982 Astraraea Felix, 1900}

Especie tipo: Thamnastraea multiradiata Reuss, 1854.

\section{Astraraea media (Sowerby, 1835)}

$$
\text { Fig. 3D }
$$

\section{?1937 Synastraea media (Sowerby); Bataller, 196.}

1982 Astraraea media (Sowerby); Beauvais, v. II, 31, lám. XXIV, fig. 4; y su sinonimia.

Material: Una colonia de hábito domal, parcialmente silicificada (UAB-32176), de les Collades de Basturs y una colonia domal (UAB-32177), de Sant Martí de Vilanoveta.

\section{Descripción}

Colonia thamnasterioide con colinas toliformes pronunciadas y cálices poligonales (tetragonales y pentagonales) con fosetas calicinales amplias. Número elevado de láminas biseptales (27-53) de sección rectangular, muy perforadas, con las caras laterales lisas y márgenes distales heterodontos y libres, observándose ocasionalmente alguna coalescencia simple. Columnilla parietal fascicular papilar de difícil delimitación debido a la discontinuidad de las láminas biseptales. Endoteca bien desarrollada, constituida por sinaptículos y un número mayor de disepimentos vesiculares finos poco arqueados. Microestructura esquelética penular, formada mayoritariamente por menianas y en menor proporción por pénulas libres con expansiones laterales de sección transversal cuadrada. Gemación extracalicinal. (Ver las dimensiones de los caracteres descritos, en la Tabla 1.)

\section{Observaciones}

Según Beauvais (1982, v. II, pág. 29) Astraraea presenta gemación intracalicinal. Como uno de los ejemplares estudiados (UAB-32172) muestra poliporitos juveniles sobre colinas que separan poliporitos adultos, precisamos que este género puede presentar ambos tipos de gemación.
SUBORDEN FUNGIINA Verril, 1865

Superfamilia THAMNASTERIOIDAE Alloiteau, 1952

Familia Andemantastraeidae Alloiteau, 1952

Neocaeniopsis Alloiteau, 1957

Especie tipo: Phyllocoenia excelsa de Fromentel, 1884.

\section{Neocaeniopsis excelsa (de Fromentel, 1867)} Fig. 5.D

1957 Neocaeniopsis excelsa (de Fromentel); Alloiteau, 127.

1982 Neocaeniopsis excelsa (de Fromentel); Beauvais, v. II, 103.

Material: Colonia de hábito tábulo-lenticular (UAB-32172), de Sant Martí de Vilanoveta.

\section{Descripción}

Colonia plocoide con cálices circulares o moderadamente elípticos, con 24 costoseptos por poliporito, distribuidos en tres ciclos. Los costoseptos son muy ensanchados, ligeramente bicuneiformes, con la región costal de igual longitud en todos los ciclos, continuos, libres y comúnmente subconfluentes, siendo no confluentes en las zonas de la periteca delimitadas por tres o cuatro poliporitos. Caras laterales con gránulos espiniformes generalmente alternos. Columnilla parietal esponjosa no siempre observable. Endoteca disepimental vesicular formada por disepimentos delgados distribuidos por todo el poliporito. Exoteca disepimental celular y muralla mixta paratecal y sinapticulotecal continua y compacta (sólo en algunos casos la muralla se presenta perforada). El contorno de los costoseptos en sección longitudinal es similar al contorno de la microestructura penular descrita y figurada por G. Gill para el género jurásico Calamosseris (Gill, 1967, lám. V, figs. 3-4) y el género triásico Tropidendron (Gill, 1982, lám. 2, fig. 5), pero no se ha podido demostrar la presencia o ausencia de pénulas en el ejemplar debido a su pobre estado de conservación. (Ver las dimensiones de los caracteres descritos, en la Tabla 1.)

\section{Observaciones}

El ejemplar estudiado se corresponde con la diagnosis de Neocaeniopsis de Alloiteau (1957, pág. 127-128). De las 7 especies que Beauvais (1982) describe de este género, sólo dos presentan un número constante de 24 elementos radiales distribuidos en tres ciclos: $N$. excelsa, especie que no figura, y N. fromenteli Beauvais, 1982. Estas dos especies se diferencian esencialmente por la presencia, respectivamente, de una columnilla parietal y una columnilla laminar, así como por tener diferentes diámetros calicinales y diferentes distancias entre centros calicinales de poliporitos contiguos. Hemos atribuido el ejemplar estudiado a $N$. excelsa puesto que presenta el mismo tipo de columnilla, aunque los valores del diámetro calicinal y de la distancia entre centros calicinales de poliporitos contiguos son significativamente inferiores a los del holotipo de esta especie y más parecidos a los de $N$. fromenteli. También aquí, como en Placocoenia decussata (ver más arriba), hemos supuesto 


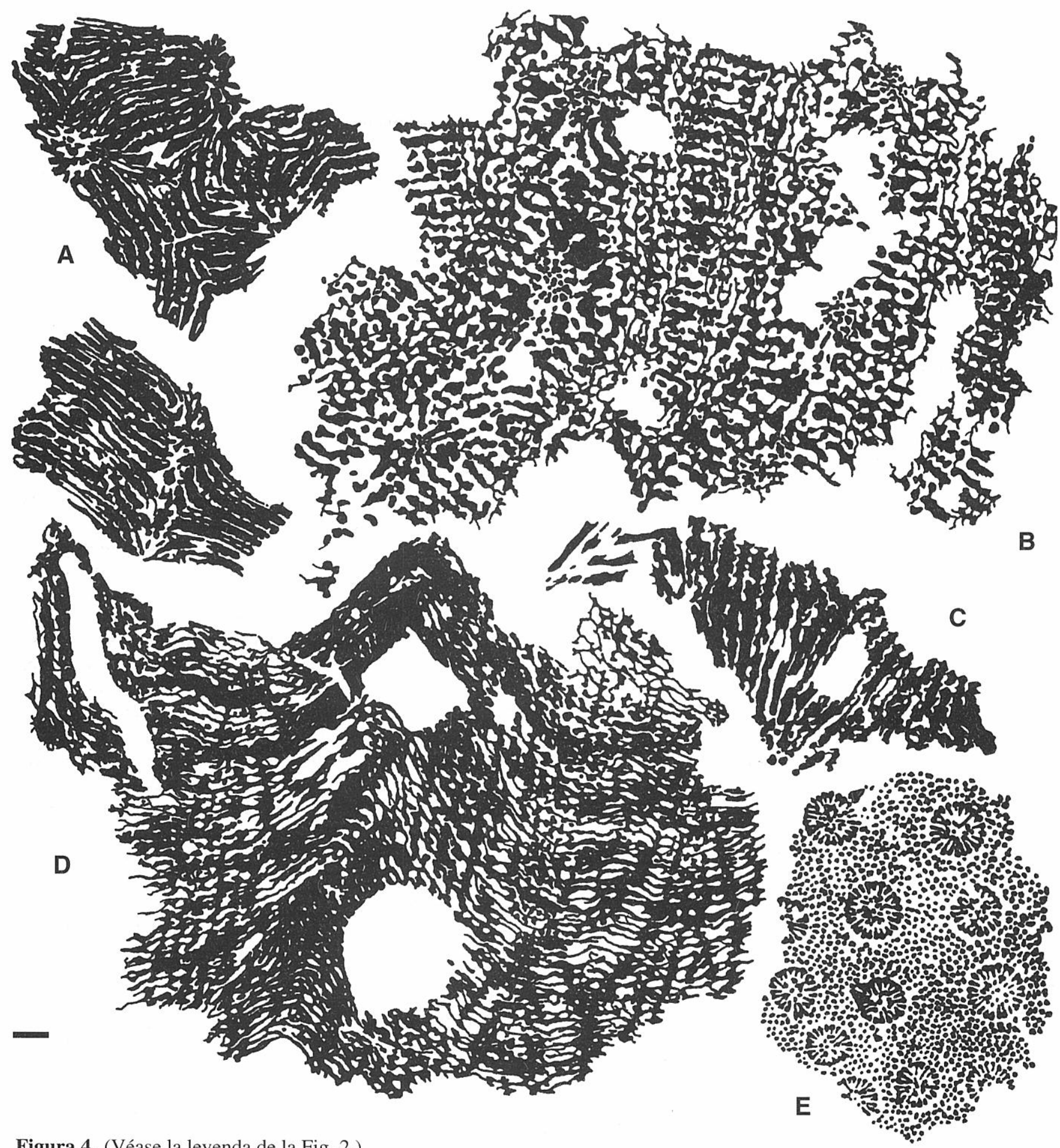

Figura 4. (Véase la leyenda de la Fig. 2.)

A. Fungiastraea sp. Ejemplar UAB-32186. Sección transversal. B. Elasmofungia comoserioides Alloiteau, 1957. Ejemplar UAB-32146. Sección transversal. Las láminas biseptales están profusa, aunque irregularmente, perforadas a lo largo de todo el eje axio-periférico. C. Corbariastraea junctiseptata (Oppenheim, 1930). Ejemplar UAB-32165. Sección transversal. D. Elasmofungia comoserioides Alloiteau, 1957. Ejemplar UAB-32147. Sección longitudinal. Se observan las típicas líneas sinusoidales formadas por los disepimentos que unen las trabéculas siguiendo las líneas de crecimiento del coral. Ejemplar procedente de les Collades de Basturs. E. Actinacis reussi Oppenheim, 1930. Ejemplar UAB-32152; colonia domal. Sección transversal. Se puede observar la anastomosis característica en $\mathrm{V}$ y en Y de los elementos radiales perforados, y la típica exoteca tábulo-columnar.

que las diferencias en las dimensiones de estos parámetros biométricos podrían ser debidas a factores ecológicos, basándonos en los resultados obtenidos por diversos autores (Foster, 1979, 1983, 1985; Budd et al., 1994; Amaral, 1994; Riegl y Piller, 1997; entre otros) sobre la variabilidad de los corales actuales y fósiles. 


\section{Neocaeniopsis fromenteli Beauvais, 1982}

Fig. 2.G

1884 Phyllocoenia lepida de Fromentel, 552, lám. 152, figs. 1-la.

*1982 Neocaeniopsis fromenteli Beauvais, 104.

Material: Dos colonias de hábito domal (UAB-32173, UAB-32174), de Sant Martí de Vilanoveta.

\section{Descripción}

Colonia plocoide, ocasionalmente cerioide, de cálices circulares con 24 costoseptos por poliporito, distribuidos en tres ciclos (los de primero y segundo ciclos de longitud muy similar). Costoseptos cuneiformes continuos, con numerosos gránulos espiniformes bastante sobresalientes y opuestos a ambos lados del costosepto. No confluentes o subconfluentes. Columnilla laminar poco desarrollada. Costoseptos contiguos unidos por algunos pocos sinaptículos en la región axial. Endoteca disepimental celular escasa. Muralla compacta, continua, paratecal y sinapticulotecal. Exoteca disepimental celular poco desarrollada y de extensión reducida. (Ver las dimensiones de los caracteres descritos, en la Tabla 1.)

Familia Thamnasteriidae Vaughan y Wells, 1943, emend. Alloiteau, 1952

Mesomorpha Pratz, 1883, emend. Felix, 1903

Especie tipo: Porites mammillata Reuss, 1854.

\section{Mesomorpha mammillata (Reuss, 1854)} Fig. 5.C

*1854 Porites mammillata Reuss, 129, lám. X, figs. 9-10.

1957 Ahrdorffia mammillata (Reuss); Alloiteau, lám. 4, fig. 10.

1957 Mesomorpha mammillata (Reuss); Alloiteau, 209.

1982 Mesomorpha mammillata (Reuss); Beauvais, v. 11, págs. 61-62, lám. XXVI, fig. 5.

Material: Colonia de hábito laminar (UAB-32148), de Sant Martí de Vilanoveta.

\section{Descripción}

Colonia thamnasterioide de poliporitos pequeños y circulares, con un número variable de láminas biseptales (>16) de sección rectangular y continuas presentando a veces poros en la región central. Presencia de gránulos redondeados, grandes y alternos que dan a las láminas biseptales un aspecto ondulado parecido, aunque no relacionado, al de la morfología en zigzag de los elementos radiales producida por la disposición alterna de sus trabéculas. Las láminas son algunas veces subconfluentes, anastomosadas regularmente y en general de dos en dos a una misma distancia del eje, prolongándose y soldándose una de las láminas a la columnilla hasta un total de 8 láminas por columnilla. Columnilla estiliforme. Abundantes sinaptículos en la región media de las láminas biseptales, sin llegar a constituir una muralla. Numerosos disepimentos celulares muy finos. (Ver las dimensiones de los caracteres descritos, en la Tabla 1.)

\section{Observaciones}

Alloiteau (1957) y Beauvais (1982) señalan la presencia de gránulos espiniformes en Mesomorpha. En el ejemplar estudiado no hemos observado este tipo de gránulos, que la recristalización puede haber enmascarado, pero los extremos de los sinaptículos tienen forma cónica, lo que podría indicar que se han formado por la unión de gránulos espiniformes.

Fungiastraea Alloiteau, 1952

Especie tipo: Astrea laganum Michelin, 1841.

\section{Fungiastraea $\mathrm{sp}$.}

Fig. 4.A

Material: Colonia de hábito laminar (UAB-32186), de Sant Martí de Vilanoveta.

\section{Descripción}

Colonia thamnasterioide con los cálices alineados en series calicinales rectas. Láminas biseptales (21 por poliporito) anchas, de sección rectangular y ligeramente sinuosas, paralelas, extendiéndose hasta el eje y curvándose suavemente en este extremo, continuas, ornamentadas por gránulos espiniformes sobresalientes muy desarrollados y opuestos a ambos lados de la lámina. Frecuentemente, los gránulos se unen formando sinaptículos regularmente distribuidos en toda la extensión de las láminas. Columnilla parietal. (Ver las dimensiones de los caracteres descritos, en la Tabla 1.)

\section{Observaciones}

Las especies de Fungiastraea descritas por Beauvais (1982) difieren unas de las otras sólo por pequeñas variaciones en algunos parámetros biométricos, tales como el diámetro de los cálices, la distancia entre centros calicinales, el número de elementos radiales por poliporito y el número de elementos radiales por $10 \mathrm{~mm}$. En el ejemplar estudiado, la distancia entre centros calicinales es semejante a la de $F$. pseudoleptophylla (Oppenheim, 1930) y F. excavata Alloiteau, 1958, mientras que el número de elementos radiales que presenta es sensiblemente inferior al de estas dos últimas especies, encontrándose, por otra parte, dentro de los márgenes de $F$. acutidens (Reuss, 1854). Por este motivo hemos optado por dejar provisionalmente el ejemplar estudiado en nomenclatura abierta.

Thamnasteria Lesauvage, 1823

Especie tipo: Thamnasteria lamourouxi Lesauvage, 1823.

Thamnasteria aff. andranomarivensis Alloiteau, 1958

Fig. 2.E 
Material: Colonia de hábito laminar (UAB-32166), de Sant Martí de Vilanoveta.

\section{Descripción}

Colonia thamnasterioide de cálices alineados, con un número variable de láminas biseptales (27-37) y láminas triseptales en la confluencia de tres poliporitos. Son láminas de sección rectangular, generalmente en forma de sigma minúscula, coalescentes (excepto dos diametralmente opuestas) formando 2 grupos de 2 elementos y 6 grupos de 4, 5 o 6 elementos, llegando así a la zona axial del poliporito un número constante de 2 láminas libres y 8 grupos. Las caras laterales de las láminas están ornamentadas por bastantes gránulos espiniformes, algunos redondeados, poco sobresalientes y dispersos. Columnilla estiliforme. Algunos sinaptículos y muy pocos disepimentos. (Ver las dimensiones de los caracteres descritos, en la Tabla 1.)

\section{Observaciones}

Los caracteres que presenta el ejemplar estudiado son muy parecidos a los de Thamnasteria andranomarivensis Alloiteau, 1958, excepto en la abundancia de disepimentos endotecales, numerosos en esta última y escasos en nuestro ejemplar, y en el número de elementos radiales por $10 \mathrm{~mm}$, sensiblemente superior en el ejemplar estudiado. Por otra parte, aunque en $T$. andranomarivensis los cálices no se presentan alineados, los elementos radiales se encuentran anastomosados siguiendo una "ley" parecida a la que siguen los del ejemplar estudiado. No obstante, dada la gran diferencia de edad entre los dos ejemplares (unos 74 millones de años) dudamos que se trate de la misma especie.

\section{SUBORDEN MICROSOLENINA Morycowa y Roniewicz, 1995 \\ Familia Synastraeidae Alloiteau, 1952, emend. Beauvais, 1982 Dimorphastraea d'Orbigny, 1849}

Especie tipo: Dimorphastraea grandiflora de Fromentel, 1857.

\section{Dimorphastraea sp. 1} Fig. 3.C

Material: Colonia de hábito laminar (UAB-32167), de Sant Martí de Vilanoveta.

\section{Descripción}

Colonia thamnasterioide de cálices dispuestos en series concéntricas. Número muy variable de láminas biseptales (comúnmente unas 26), distribuidas en dos ciclos (láminas de primer ciclo más anchas que las del segundo). Son láminas bicuneiformes (regiones septales ensanchadas) y escasamente perforadas en el margen axial. Las caras laterales presentan gránulos espiniformes opuestos. Las láminas forman uniones triples, generando láminas triseptales en la confluencia de tres poliporitos contiguos y son coalescentes con anastomosis irregular simple o múltiple (hasta 4 elementos). Generalmente, la coalescencia se produce por la unión del margen axial de las láminas del segundo ciclo a la cara lateral de las del primero. Columnilla parietal fascicular poco desarrollada, rodeada por los márgenes axiales de las láminas, individualizados por poros verticales, que en algunos casos pueden parecer papilas de la columnilla o una corona de seis pali. Sinaptículos poco numerosos, situados principalmente en la zona axial del poliporito. Numerosos disepimentos planos en la zona periférica, sin llegar a formar muralla. (Ver las dimensiones de los caracteres descritos, en la Tabla 1.)

\section{Observaciones}

Beauvais (1982) utiliza tres criterios principales para la distinción de especies en Dimorphastraea: (1) presencia o ausencia de colinas toliformes, (2) presencia o ausencia de un poliporito central más desarrollado (poliporito del pólipo padre) y (3) número de elementos radiales por 10 $\mathrm{mm}$ en el margen calicinal del poliporito central. Dado que estos caracteres no han podido ser observados en el ejemplar estudiado, por su mal estado de conservación, hemos dejado la atribución en nomenclatura abierta.

\section{Dimorphastraea sp. 2}

Fig. 5.B

Material: Dos ejemplares (UAB-32159, UAB-32160), de Sant Martí de Vilanoveta.

\section{Descripción}

Colonia thamnasterioide de cálices dispuestos en series concéntricas. Número elevado de láminas biseptales (20-28) sin una clara distribución en ciclos. Son láminas de sección rectangular, perforadas por poros horizontales (estructura penular) en toda su extensión, pero especialmente en la zona axial. Caras laterales ornamentadas por numerosos gránulos espiniformes, distribuidos regularmente y opuestos a ambos lados de la lámina. Láminas biseptales libres, aunque en algunos casos se observan dos láminas unidas por un sinaptículo en su margen axial. La perforación que presentan las láminas dificulta la interpretación de las estructuras axiales, sin embargo se ha podido observar una columnilla parietal fascicular poco desarrollada, rodeada por los márgenes axiales de las láminas individualizados por poros. Los sinaptículos son muy poco abundantes, pero los disepimentos, principalmente celulares y muy finos, son más comunes, concentrándose en la región media de las láminas biseptales, pero sin llegar a constituir una muralla. En sección longitudinal, se observa que los disepimentos crecen a partir de gránulos. (Ver las dimensiones de los caracteres descritos, en la Tabla 1.)

\section{Observaciones}

Hemos dejado la especie en nomenclatura abierta dado que la pobre conservación de los ejemplares no nos ha permitido observar los caracteres diagnósticos utilizados por Beauvais (1982).

Valliculastraea Alloiteau, 1957

Especie tipo: Valliculastraea jauberti Alloiteau, 1957. 


\section{Valliculastraea spinosa (Oppenheim, 1930)} Fig. 5.A

*1930 Dimorphastraea spinosa Oppenheim, 193, lám. XXIV, figs. 6-6a.

1982 Valliculastraea spinosa (Oppenheim); Beauvais, v. II, 131.

Material: Tres colonias, una de hábito domal (UAB-32153), otra columnar (UAB-32154) y otra laminar (UAB-32163), de les Collades de Basturs; y dos colonias laminares (UAB32161, UAB32162), de Sant Martí de Vilanoveta.

\section{Descripción}

Colonia thamnasterioide de cálices circulares o poligonales (generalmente hexagonales), delimitados por colinas toliformes y un número variable pero siempre elevado de láminas biseptales (generalmente $>36$ ) sin una clara distribución en ciclos. Microestructura penular formada por menianas, bien visibles en el ejemplar UAB-32153. Láminas biseptales de sección rectangular, con algunos poros principalmente en la región próxima al margen axial. Caras laterales ornamentadas por numerosos gránulos espiniformes bastante sobresalientes, dispuestos paralelamente al margen distal y unidos formando estrías continuas, y mayoritariamente opuestos a ambos lados de la lámina. Las láminas biseptales presentan coalescencias irregulares dobles (ocasionalmente cuádruples) y no más de 2-3 coalescencias por poliporito. Las coalescencias tienen lugar entre láminas cortas y láminas más largas por medio de sinaptículos que unen el margen axial de las primeras con las caras laterales de las segundas. Columnilla parietal fascicular chicoriacia. Sinaptículos numerosos especialmente en la región media de las láminas pero sin llegar a formar muralla. Disepimentos celulares escasos e irregularmente distribuidos. Gemación intracalicinal y extracalicinal generando principalmente cálices aislados, pero también cálices alineados en series de 2-3 poliporitos. (Ver las dimensiones de los caracteres descritos, en la Tabla 1.)

\section{Observaciones}

Los ejemplares estudiados se diferencian del holotipo de Valliculastraea spinosa por presentar un número superior de elementos radiales por $10 \mathrm{~mm}$.

\section{Valliculastraea texta (Oppenheim, 1930)}

\section{Fig. 3.1}

*1930 Maeandraraea texta Oppenheim, 212, lám. XVI, fig. 5. 1982 Valliculastraea texta (Oppenheim); Beauvais, v. II, 132, lám. XXXIX, fig. 4.

Material: Colonia de hábito laminar (UAB-32157), de Sant Martí de Vilanoveta.

\section{Descripción.}

Colonia thamnasterioide de cálices circulares con un número variable y elevado (31-52) de láminas biseptales sin distribución aparente en ciclos. Son láminas de sección rectangular e irregularmente perforadas, especialmente en la región axial. Caras laterales ornamentadas por gránulos espiniformes alternos y opuestos a ambos lados de la lámina. Láminas biseptales libres pero algunas veces con coalescencias simples o triples de distribución irregular. Columnilla parietal fascicular que parece una columnilla parietal rodeada de una corona de pali. Sinaptículos numerosos en la región central de las láminas biseptales. Sin muralla. Disepimentos también numerosos, celulares y pequeños en todo el lumen. (Ver las dimensiones de los caracteres descritos, en la Tabla 1.)

\section{Observaciones}

Aunque las pequeñas dimensiones del ejemplar no permiten observar la disposición de los cálices, el resto de caracteres coinciden con los de Valliculastraea texta.

Familia Latomaeandridae Alloiteau, 1952, emend. Beauvais, 1982 Brachyseris Alloiteau, 1947

Especie tipo: Latomaeandra morchella Reuss, 1854.

\section{Brachyseris tenerrima (Oppenheim, 1930)} Fig. 2.F

1982 Brachyseris tenerrima (Oppenheim); Beauvais, v. II, 240; y su sinonimia.

Material: Dos colonias de hábito domal (UAB-32184, UAB-32185), de Sant Martí de Vilanoveta.

\section{Descripción}

Colonia thamnasterioide con cálices dispuestos en alineaciones mal definidas y un número elevado (21-32) de láminas biseptales de sección rectangular, poco perforadas y con las caras laterales ornamentadas por gránulos espiniformes bien desarrollados, alternos y opuestos a ambos lados de la lámina, en una misma proporción. En muchos casos, los gránulos crecen desmesuradamente hacia la cavidad interseptal, llegando alguna vez a formar sinaptículos, confiriendo a las láminas su aspecto espinoso característico. Microestructura penular con pénulas libres y hemipénulas. Las láminas son coalescentes con anastomosis irregular simple y en algunos casos triple. Columnilla parietal fascicular poco definida. Sinaptículos anchos, más frecuentes en la región intermedia de las láminas. Sin muralla. Disepimentos celulares muy finos y muy numerosos, uniendo las láminas biseptales tanto por los gránulos como por otras zonas y distribuidos regularmente por todo el lumen a excepción de la región axial. (Ver las dimensiones de los caracteres descritos, en la Tabla 1.)

Elasmofungia Alloiteau, 1957

Especie tipo: Elasmofungia comoserioides Alloiteau, 1957.

\section{Elasmofungia comoserioides Alloiteau, 1957} Figs. 4.B; 4.D

*1957 Elasmofungia comoserioides Alloiteau, 286, lám. 16, fig. 4; lám. 17, fig. 4.

.v 1980 Elasmofungia cosmoserioides Alloiteau; Vidal, 58, lám. IX, figs. 16-18. 

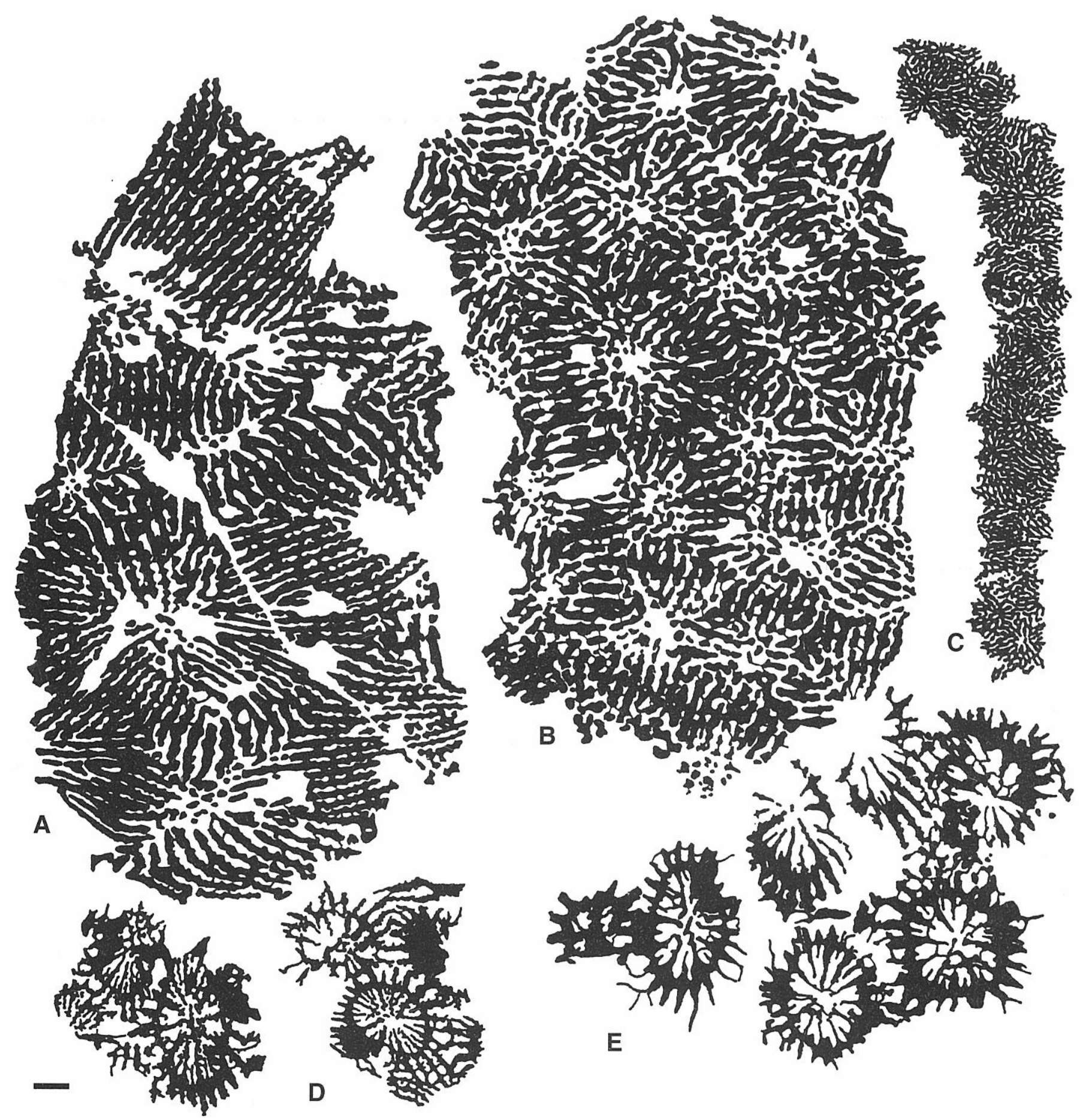

Figura 5. (Véase la leyenda de la Fig. 2.)

A. Valliculastraea spinosa (Oppenheim, 1930). Ejemplar UAB-32163. Sección transversal. Ejemplar procedente de les Collades de Basturs. B. Dimorphastraea sp. 2. Ejemplar UAB-32160. Sección transversal. C. Mesomorpha mammillata (Reuss, 1854). Ejemplar UAB-32148. Sección transversal. D. Neocaeniopsis excelsa (de Fromentel, 1867). Ejemplar UAB-32172. Sección transversal. E. Placocoenia dumortieri de Fromentel, 1879. Ejemplar UAB32155. Sección transversal.

1995 Summiktaraea concentrica Reuss; Darrell et al., 61.

.v 1995 Summiktaraea concentrica Reuss; Valldeperas y Rosen, 182, fig. 2(2).

Material: Se han estudiado 18 ejemplares procedentes de Sant Martí de Vilanoveta y uno de les Collades de Basturs.
Los caracteres externos se han analizado en detalle en 7 ejemplares de hábito laminar que conservan la superficie calicinal, y los internos, en 12 ejemplares laminares y tábulo-lenticulares que no conservan la superficie externa. Han sido figurados los ejemplares UAB-32146, UAB$32147, \mathrm{UAB}-32188$. 


\section{Descripción}

Colonia thamnasterioide con los cálices dispuestos en series largas (de $17 \mathrm{~cm}$ de longitud máxima y hasta 20 poliporitos por serie), concéntricas y separadas por colinas tectiformes, con un número pequeño (16-18) de láminas biseptales dispuestas paralelamente entre sí a lo largo de las series. Microestructura penular formada por pénulas libres bien desarrolladas, con las expansiones laterales ligeramente arqueadas hacia la región distal. Las láminas biseptales tienen sección rectangular y están muy perforadas, con las caras laterales ornamentadas por gránulos espiniformes opuestos a cada cara, que corresponden a expansiones penulares, enlazados por disepimentos largos a través de las cavidades interseptales. Son láminas libres. Columnilla parietal fascicular papilar, fácil de confundir con el borde axial de los elementos radiales. Algún sinaptículo y numerosos disepimentos vesiculares convexos, especialmente en la región central de los elementos radiales. Sin muralla (como máximo una muralla paratecal perforada y discontinua, muy poco definida, separando series calicinales). Los disepimentos se unen formando curvas sinuosas muy características, paralelas a las líneas de crecimiento del coral. Presencia de una holoteca fina, a veces discontinua. (Ver las dimensiones de los caracteres descritos, en la Tabla 1.)

\section{Observaciones}

Asunción Vidal (1980) describe y figura dos ejemplares procedentes de les Collades de Basturs, que atribuye a Elasmofungia comoserioides y cuyas características coinciden con las de los ejemplares estudiados. Tanto unos como otros difieren de Elasmofungia Alloiteau, 1957 por el tipo de elementos radiales, la ausencia de muralla y la presencia de una columnilla parietal fascicular papilar bien desarrollada (Alloiteau describe costoseptos, una muralla sinapticulotecal y una columnilla parietal rudimentaria). Por otra parte, Darrell et al. (1995), en un estudio taxonómico preliminar de los corales de les Collades de Basturs, y Valldeperas y Rosen (1995), en un estudio taxonómico preliminar de los corales de Sant Martí de Vilanoveta, atribuyen los ejemplares que hemos estudiado a Summiktaraea concentrica (Reuss, 1854). En este último trabajo, se señala que estos dos géneros (Familia Latomaeandridae) son muy parecidos y los autores no descartan la posibilidad de que sean sinónimos. Además, Meandrarea tenerrima Oppenheim, tal como señala Alloiteau (1957), presenta las mismas formaciones disepimentales sinuosas que Elasmofungia comoserioides, pero Alloiteau $(1952,1957)$, argumentando la regularidad de las perforaciones de los elementos radiales, sitúa $M$. tenerrima en la Familia Microsolenidae. En un trabajo reciente, Morycowa y Roniewicz (1995) crean un nuevo suborden (Microsolenina) en el que sitúan, entre otras, a las familias Microsolenidae y Latomaeandridae, siendo las diferencias principales entre ellas la presencia de elementos radiales regularmente perforados en la primera, y de elementos radiales compactos o regularmente perforados en la región distal o distal-interna en la segunda. Del estudio de los ejemplares de les Collades de
Basturs y de Sant Martí de Vilanoveta se concluye que estas características no son totalmente excluyentes, estando dichos ejemplares a caballo de las dos diagnosis. Dado que no realizamos una revisión formal de Elasmofungia, Summiktaraea y Meandrarea, hemos optado por conservar la determinación original de Elasmofungia comoserioides de Asunción Vidal (1980) para los ejemplares estudiados.

\section{SUBORDEN FAVIINA Vaughan y Wells, 1943 \\ Familia Trachyphylliidae Wells, 1956 \\ Columastrea d'Orbigny, 1849}

Especie tipo: Astrea striata Goldfuss, 1826.

\section{Columastrea $\mathrm{sp}$.}

Fig. 2.B

Material: Un fragmento pequeño de colonia masiva (UAB32169), de les Collades de Basturs, y un fragmento"de colonia masiva (UAB-32168) cuya total silicificación permite observar el detalle de la superficie calicinal y dos colonias de hábito laminar (UAB-32170, UAB-32171), de Sant Martí de Vilanoveta.

\section{Descripción}

Colonia plocoide, parcialmente cerioide, de cálices circulares, con márgenes poco sobresalientes pero bien delimitados y foseta calicinal medianamente profunda y ancha. Presencia de 24 costoseptos por poliporito distribuidos en tres ciclos, los de primero y segundo ciclos casi de la misma longitud. Son costoseptos continuos, ensanchados, claviformes (excepto los del tercero) o ligeramente bicuneiformes. Caras laterales pobremente ornamentadas por gránulos espiniformes de diferentes tamaños y alternos. Los costoseptos son libres y no confluentes, excepto en aquellas áreas donde la extensión de la periteca es pequeña, que pueden ser subconfluentes e incluso confluentes. Columnilla estiliforme irregular, rodeada de una corona de 6 pali situados delante de los elementos radiales de segundo ciclo. Endoteca disepimental celular, con un número mayor de disepimentos en la zona axial del poliporito, uniendo regiones septales de costoseptos contiguos, costoseptos con pali, pali con pali y los pali con la columnilla. Cuando la endoteca está bien desarrollada, puede desfigurar la corona de pali. Muralla septotecal por ensanchamiento de la región costal de los costoseptos en la región proximal de los poliporitos y, en algunos casos, paratecal en la región distal, continua y generalmente compacta. Exoteca disepimental celular pequeña. Gemación extracalicinal. (Ver las dimensiones de los caracteres descritos, en la Tabla 1.)

\section{Observaciones}

Los caracteres de los ejemplares estudiados se ajustan a la diagnosis de Columastrea (Alloiteau, 1957) y coinciden con los caracteres que Beauvais (1982) atribuye a Columastrea leymeriei Vidal. Sin embargo, los 
caracteres de nuestros ejemplares no coinciden con los que hemos observado en los topotipos de esta especie.

\section{SUBORDEN MEANDRIINA Alloiteau, 1952 Familia Meandrinidae Gray, 1847 Meandroria Alloiteau, 1952}

Especie tipo: Meandrina radiata Michelin, 1847.

\section{Meandroria tenella (Goldfuss, 1826)} Fig. 3.B

*1826 Meandrina tenella Goldfuss, v. I, 63, lám. XXI, fig. 4. 1980 Meandroria konincki (Milne-Edwards y Haime); Vidal, 47, lám. XII, figs. 1, 2.

1982 Meandroria tenella (Goldfuss); Beauvais, v. I, 210, lám. XVIII, figs. 2, 3; lám. XIX, fig. 1.

Material: Un fragmento de colonia de hábito laminar parcialmente silicificada (UAB-32149), de les Collades de Basturs y dos ejemplares (UAB-32150, UAB-32151), de Sant Martí de Vilanoveta.

\section{Descripción}

Colonia meandroide de series calicinales medianamente largas $(>36 \mathrm{~mm})$, rectas o ligerísimamente onduladas, delimitadas por colinas toliformes, con un pequeño surco en la cresta o tectiformes, formadas por la unión directa de las murallas. Centros calicinales reconocibles por la presencia de una columnilla estiliforme, algunas veces ligeramente alargada (laminar). Tres ciclos de costoseptos, los de primer ciclo son ropaloides y ensanchados, los de segundo y tercero tienen sección rectangular o son claviformes. La región costal es muy pequeña, visible sólo en la superficie basal del polípero. Los costoseptos ropaloides presentan la "T" asimétrica, siendo el brazo más largo el más próximo a la columnilla. Los costoseptos son continuos y presentan numerosos gránulos redondeados y poco sobresalientes, aislados y alternos, a veces dispersos pero más comúnmente dispuestos paralelamente al margen distal de la región septal, confluentes (alguna vez subconfluentes) o libres. Presencia de septos de valle con numerosas estrías. Muralla septotecal, por ensanchamiento del margen costal externo de los costoseptos, compacta y continua. Endoteca celular formada por disepimentos muy finos, más densa en el margen axial de los costoseptos. (Ver las dimensiones de los caracteres descritos, en la Tabla 1.)

\section{Observaciones}

Dos de los ejemplares estudiados presentan, como consecuencia de la silicificación, un gran ensanchamiento de los elementos radiales de primero y segundo ciclos que dificulta su diferenciación; en algunas zonas de la colonia, sin embargo, se pueden apreciar claramente los tres ciclos de costoseptos característicos de Meandroria (Alloiteau, 1957, pág. 169). Es de señalar que la dificultad de reconocer, en muestra de mano, el tercer ciclo y de distinguir, en sección pulida, el primero y segundo ciclos, cuando los ejemplares están silicificados, ha llevado a algunos autores (Vidal, 1980; Beauvais, 1982) a describir dos únicos ciclos en Meandroria.

\section{AFINIDADES CON OTRAS ÁREAS}

La gran mayoría de especies que aparecen en Sant Martí de Vilanoveta fueron originariamente descritas en el Cretácico Superior del Grupo Gosau y depósitos afines en los Alpes calcáreos (ver Alloiteau, 1957; Beauvais, 1982), pero su riqueza es muy inferior a la de aquella zona alpina. Así, la fauna coralina de edad ConiacienseSantoniense superior descrita por Beauvais, presenta un número de especies mucho mayor que la de Sant Martí de Vilanoveta (224 y 21, respectivamente), coincidiendo 16 géneros y 10 especies. Por otro lado, aunque la asociación de Sant Martí de Vilanoveta presenta una riqueza semejante a algunas asociaciones de corales procedentes de aquella zona, su fauna es notablemente diferente. Así, coincide sólo en 4 géneros y dos especies con la fauna de la Región de Gosau descrita por Alloiteau (1941), y no coincide en ninguna especie y sólo en 4 géneros con la asociación procedente del Grupo Gosau en Brandenberg, de edad Turoniense-Coniaciense, reseñada por Sanders y Baron-Szabo (1997). Por otro lado, la fauna coralina de Sant Martí de Vilanoveta presenta algunas afinidades, pero también diferencias importantes, con la fauna de otras localidades del Cretácico Superior. Así, aun cuando presenta un número de especies comparable a la fauna de edad CenomanienseCampaniense medio de la montaña de la Maiella en los Apeninos centrales (Stössel, 1997), a la del Coniaciense de Madagascar (Ankotrofotsy) (Alloiteau, 1958) y a la identificada en el Santoniense superior de FiguièresAnténore (Provence, Francia) por Beauvais (1982), ninguna especie y sólo un máximo de 5 géneros coinciden entre aquella y estas localidades. Respecto a la fauna de edad Coniaciense-Campaniense de Corbières en los Pirineos orientales, descrita por Beauvais (1982), ésta presenta un número de especies mucho mayor (168 y 21 , respectivamente) y coinciden 16 géneros y 10 especies. Las diferencias mencionadas pueden ser debidas a factores temporales, paleogeográficos y/o ecológicos, supuestos éstos que por el momento no pueden ser aseverados. Para afirmarlos sería preciso una revisión profunda de la taxonomía de los corales del Cretácico Superior, combinada con la revisión taxonómica y bioestratigráfica del material hasta ahora identificado. Finalmente, cabe señalar que la asociación de corales de Sant Martí de Vilanoveta es remarcablemente diferente a la de les Collades de Basturs descrita por Vidal (1980) a pesar de su proximidad temporal y geográfica. El número de especies en Sant Martí de Vilanoveta es inferior al de les Collades de Basturs (21 y 33, respectivamente) y sólo 4 géneros y dos especies son comunes a ambas asociaciones. Esta disimilitud puede reflejar importantes diferencias en las condiciones ambientales de los ejemplares estudiados. Esta suposición estaría fundamentada en que la mayoría de ejemplares recogidos en Sant Martí de Vilanoveta proceden de materiales calcáreos, calizas y rocas calizo-margosas, mientras que los de les Collades de Basturs fueron mayoritariamente extraídos de materiales margosos. 


\section{CONCLUSIONES}

Del análisis bibliográfico se deduce que la taxonomía de los corales escleractinios se ve muy complicada por la dificultad de distinguir especies claramente. Como invertebrados marinos sésiles, los corales presentan una gran variación morfológica en respuesta a factores ambientales (ver Foster, 1979, 1985), a la vez que pueden también presentar un alto polimorfismo genético (ver, por ejemplo, Amaral, 1994). Los resultados obtenidos por diversos autores (Foster, 1979, 1983, 1985; Budd et al., 1994; Amaral, 1994; Riegl y Piller, 1997; entre otros) sobre la variabilidad de los corales actuales y fósiles indican que: 1) todos los caracteres morfológicos de las especies coralinas pueden variar extensamente, siendo esta variación diferente entre especies y, 2) existen pocos caracteres estables para definir especies. Esta amplia variabilidad, en respuesta directa al ambiente o por diferenciación genética o por ambas cosas, de los caracteres que se han utilizado, típicamente, para distinguir géneros y separar especies en los géneros, es la responsable de la complicada e inestable taxonomía de los corales.

El trato que reciben los corales escleractinios del Cretácico Superior, en las monografías taxonómicas pertinentes, está anticuado y es inadecuado para definir taxones, ya que raras veces tiene en cuenta las importantes implicaciones taxonómicas de la variabilidad de los caracteres utilizados. Así, en el análisis taxonómico de los corales de Sant Martí de Vilanoveta, hemos observado que, en algunos casos, las morfologías de las especies se solapan y que a algunas especies se les han dado diferentes nombres porque presentaban pequeñas diferencias morfológicas. En este trabajo, las especies han estado identificadas siguiendo un análisis taxonómico clásico, como primer paso a una subsiguiente revisión profunda de la taxonomía de los corales escleractinios del Cretácico Superior.

\section{AGRADECIMIENTOS}

Los autores agradecen a Peter W. Skelton, Pere Busquets y a la editora María Luisa Martínez-Chacón, sus útiles comentarios y sugerencias al manuscrito original. Del mismo modo, FXV expresa su más sincero agradecimiento a Germán Álvarez por sus consejos y discusiones en torno a la taxonomía, y la lectura crítica de un manuscrito anterior; y a Dani Gutiérrez por su contagioso entusiasmo y colaboración en el estudio de los corales. El trabajo ha sido subvencionado parcialmente por el Proyecto de Investigación de la DGICYT n ${ }^{\circ}$ PB94-0683.

\section{BIBLIOGRAFÍA}

Alloiteau, J. 1941. Révision de la collection H. Michelin. Polipiers d'Anthozoaires fossiles. I. Crétacé. Mémoires du Muséum National d'Histoire Naturelle, n.s., v. XVI, 1, 1-100.
Alloiteau, J. 1952. Embranchement des coelentérés. In: Traité de Paléontologie (Ed. J. Piveteau), v. I, Masson, 376-684.

Alloiteau, J. 1954. Le genre Actinastrea d'Orbigny 1849 dans le Crétacé supérieur français. Annales Hebert et Haug, 4(8), 1-104.

Alloiteau, J. 1957. Contribution à la systématique des Madréporaires fossiles. Centre National de Recherche Scientifique, París, 2 vols., 462 pp.

Alloiteau, J. 1958. Monographie des Madréporaires fossiles de Madagascar. Annales géologiques de Madagascar, 25, 1-218.

Amaral, F. D. 1994. Morphological variation in the reef coral Montastrea cavernosa in Brazil. Coral Reefs, 13, 113-117.

Bataller, J. R. 1937. La fauna coral'lina del Cretàcic de Catalunya i regions limítrofes. Arxius de I'Escola Superior d'Agricultura, (N.S.), (3), 1, 3-299.

Bataller, J. R. 1945. Segundo suplemento a "La fauna coral 'lina del Cretàcic de Catalunya i regions limítrofes". Anales de la Escuela de Peritos Agrícolas y Superior de Agricultura y de los Servicios Técnicos de Agricultura, 5, 3-58.

Beauvais, L. 1980. Sur la taxinomie des madréporaires mésozoïques. Acta Palaeontologica Polonica, 25 (3-4), 345-360.

Beauvais, M. 1982. Révision Systématique des Madréporaires des Couches de Gosau (Crétacé supérieur, Autriche). 5 vols. Travaux du Laboratoire de Paléontologie des lnvertébrés. Université Pierre et Marie Curie, París, 710 pp.

Budd, A. F., Johnson, K. and Potts, D. C. 1994. Recognizing morphospecies in colonial reef corals: I. Landmark-based methods. Palaeobiology, 20, 484-505.

Darrell, J. G., Gili, E., Rosen, B. R., Skelton, P. W. and Valldeperas, F. X. 1995. Les Collades de Basturs, Tremp area: Rudist and Coral formations. In: Bioconstructions of the Eocene South Pyrenean foreland basin (Vic and Igualada areas) and of the Upper Cretaceous South Central Pyrenees (Tremp area). Field Trip C - Guide Book (Eds. A. Perejón and P. Busquets). VII International Symposium on Fossil Cnidaria and Porifera, 61-63.

Foster, A. B. 1979. Environmental variation in a fossil scleractinian coral. Lethaia, 12, 245-264.

Foster, A. B. 1983. The species concept in fossil hermatipic corals: a stadistical aproach. Paleontographica Americana, 54, 58-69.

Foster, A. B. 1985. Variation within coral colonies and its importance for interpreting fossil species. Journal of Paleontology, 59, 1359-1381.

Fromentel, E. de. 1862-87. Zoophytes (11). In: Paléontologie française. Terrain crétacé. A. (Ed. d'Orbigny), Masson, 8, 624 pp.

Gili, E. 1984. Interaccions sedimentològiques i biològiques a les formacions calcàries de rudistes (Bivalvia) de les Collades de Basturs (Cretaci superior, zona sudpirinenca central). Resum de Tesi Doctoral. Publicacions de la Universitat Autònoma de Barcelona, Bellaterra, $42 \mathrm{pp}$. 
Gili, E. 1992. Palaeoecological significance of rudist constructions: a case study from les Collades de Basturs (Upper Cretaceous, South Central Pyrenees). Geologica Romana, 28, 319-325.

Gili, E., Skelton, P. W., Vicens, E. and Obrador, A. 1995. Corals to rudists - an environmentally induced assemblage sequence. In: Palaeoenvironmental models for the benthic associations of Tethyan Cretaceous carbonate platforms (Eds. J. Philip and P. W. Skelton). Palaeogeography, Palaeoclimatology, Palaeoecology, 119, 127-136.

Gili, E., Vicens, E., Obrador, A., Skelton, P. W. y López, G. 1996. Las formaciones de rudistas de la plataforma de Sant Corneli (Cretácico superior, unidad central surpirenaica). Revista Española de Paleontología, $\mathbf{N}^{\circ}$ Extraordinario, 172-181.

Gill, G. A. 1967. Quelques précisions sur les septes perforés des polypiers mésozoïques. Mémoires de la Société Géologique de France, 106, 56-82.

Gill, G. A. 1982. A supposed rhythmic mechanical process in coral skeletal growth. In: Palaeontology, Essential of Historical Geology. Proceedings of 1st International Meeting of Palaeontology, Venice, 2-4 June 1981, 445466.

Goldfuss, A. 1826-1833. Petrefacta germaniae (1). Arnz Düsseldorf, $252 \mathrm{pp}$.

Masse, J. P. and Philip, J. 1981. Cretaceous coral-rudistid buildups of France. In: European fossil reef models (Ed. D.F. Toomey). Society of Economic Paleontologists and Mineralogists, Tulsa, 30, 399-426.

Morycowa, E. and Ronievicz, E. 1995. Microstructural disparity between Recent fungiine and Mesozoic microsolenine scleractinians. Acta Palaeontologica Polonica, 40, 361-385.

Oppenheim, L. P. 1930. Die Anthozoen der Gosauschichten in den Ostalpen. Berlín, 604 pp.

Reuss, A. E. 1854. Beiträge zur charakteristik der kreideschichten in den Ostalpen, besonders im Gosauthale und am Wolfgangsee. Denkschriften der Akademie der Wissenschaften, Mathemathischnaturwissenschaftliche Klasse, 7, 157 pp.

Riegl, B. and Piller, W. E. 1997. Intra-colony variability of calice characteristics in recent Porites lutea. Boletín de la Real Sociedad Española de Historia Natural, 91, 305316.

Rosen, B. R. (en prensa). Corals, reefs, algal simbiosis and global change: the Lazarus factor. In: Biotic response to global change: the last 145 million years (Eds. S. J. Culver and P. F. Rawson), Chapman and Hall, London.
Ross, D. 1989. Facies analysis and diagenesis of Tethyan rudist reefs complexes. Ph. D. Thesis, University of Wales, Cardiff, 206 pp.

Sanders, D. and Baron-Szabo, R. C. 1997. Coral-rudist bioconstructions in the Upper Cretaceous Haidach section (Gosau Group; Northern Calcareous Alps, Austria). Facies, 36, 69-90.

Skelton, P. W., Gili, E., Vicens, E. and Obrador, A. 1995. The growth fabric of gregarious rudist elevators (hippuritids) in a Santonian carbonate platform in the southern Central Pyrenees. In: Palaeoenvironmental models for the benthic associations of Tethyan Cretaceous carbonate platforms (Eds. J. Philip and P. W. Skelton). Palaeogeography, Palaeoclimatology, Palaeoecology, 119, 107-126.

Skelton, P. W., Gili, E., Rosen, B. R. and Valldeperas, F. X. 1997. Corals and rudists in the Late Cretaceous: a critique of the hypothesis of competitive displacement. Boletín de la Real Sociedad Española de Historia Natural, 92, 223-237.

Stössel, I. 1997. Rudists and platform evolution: the Upper Cretaceous Maiella platform margin, Abruzzi, ltaly. Ph. D. Thesis. Geology Institute, Zürich, $131 \mathrm{pp.}$

Valldeperas, F. X. 1997. Estudi taxonòmic, paleoecològic $i$ estratigràfic de la biofàcies de coralls plans de l'anticlinal de Sant Corneli (Cretaci superior, unitat sudpirinenca central). Tesis de 3er Ciclo. Universitat Autònoma de Barcelona, Bellaterra, 292 + XXIX pp.

Valldeperas, F. X. y Rosen, B. R. 1995. Asociación de corales planos en la plataforma calcárea del flanco norte de Sant Corneli (Cretácico superior, unidad central surpirenaica): caracterizaciones taxonómica y paleoecológica preliminares. In: XI Jornadas de Paleontología (Eds. G. López, A. Obrador y E. Vicens), 181-184.

Veron, J. E. N. 1995. Corals in space and time: biogeography and evolution of the Scleractinia. University of South Wales Press, $321 \mathrm{pp}$.

Veron, J. E. N., Odorico, D. M., Chen, C. A. and Miller, D. J. 1996. Reassessing evolutionary relationships of scleractinian corals. Coral reefs, 15, 1-9.

Vidal, A. 1980. Los Scleractinia de Collades de Bastús (Con.-Sant., Prepirineo de la provincia de Lérida). Publicaciones de Geología de la Universidad Autónoma de Barcelona, 11, 94 pp.

Wells, J. W. 1956. Scleractinia. In: Treatise on Invertebrate Paleontology (Ed. R. C. Moore). Pt. F: Coelenterata. Geological Society of America and University of Kansas Press, 328-444. 